ainsi qu'il résulte du travail de WoxtkewITsch dans le Bulletin de la Station Bactériologique agricole, 1916, $\mathrm{n}^{\circ} 22$. Quelle forme d'albumine est, pour eux, la plus assimilable ? Voilà un point qui reste encore inéclairci et on doit se borner, pour l'instant, à supposer qu'un chauffage de l'albumine à une température élevée la rend plus facilement attaquable par les microbes.

\title{
CONTRIBUTION \\ A L'ÉTUdE DE LA LACTATION HUMAINE
}

par

Mme C. VINCENT et

Chef des travaux

M. J. VIAL

Assistant

du Laboratoire de Physiologie de la Faculté de Médecine de Lyon.

\section{DIFFICULTÉS DE LA RECHERCHE.}

10 Difficultés de prélèvement. - a) Rien ne saurait mieux résumer ces difficultés que la phrase suivante de M. Ch. Poroher, dont la compétence en matière de chimie du lait est incontestée :

"Lorsqu'il s'agit d'étudier les variations chimiques dans la sécrétion lactée de la femme, une difficulté se présente que nous n'avons pas jusqu'ici rencontrée : c'est l'impossibilité, dans l'espèce humaine, d'avoir un échantillon réellement moyen. "

Et il ajoute :

"L'échantillonnage est fort difficile et, conséquemment, les auteurs qui se sont occupés de la question ont opéré dans des conditions qu'il est toujours possible de discuter dès l'instant qu'on prend comme termes de comparaison, celles dans lesquelles on fait les prélèvements chez la vache. ")

b). Bien qu'une foule nombreuse d'auteurs aient envisagé, soit au point de vue clinique, soit au point de vue analytique, tel ou tel élément comparé du lait, ils ne l'ont fait, en général, qu'en passant et sans chercher à élargir le champ limité et souvent occasionnel de leurs recherches.

Quelques-uns, cependant, ont voulu donner une portée plus générale à ces observations; MM. PLAUCHU et RENDU ont fourni les moyennes journalières du taux butyreux du lait d'une nourrice observée du 15 décembre au 20 février, soit pendant plus de 2 mois.

Leurs conditions d'étude, quoique déjà sévères, se ressentaient cependant de la difficulté signalée par M. PoRCHer. Mais déjà leurs travaux mettaient bien en évidence l'extrême variabilité du taux butyreux journalier chez le même sujet, Cette variabilité a été encore confirmée par les travaux de MM. Denis et Talbor, par 
ceux de Young, de Richemond, de Eineman, etc. Mais tous se sont heurtés à des difficultés d'ordre pratique. Celles-ci avaient déjà découragé bien des chercheurs et gêné gravement les autres.

c) Ces difficultés sont, en effet, de plusieurs ordres :

En effet, la condition essentielle de la recherche est d'obtenir une série continue d'échantillons, recueillis dans des conditions de sévérité si absolue, qu'ils représentent sans conteste le lait total sécrété.

Il faut, en outre, pour permettre les comparaisons, connaître d'une façon précise et indiscutable la quantité de lait fournie par chaque tétée ou mulsion.

Les expérimentateurs devront pouvoir contrôler eux-mêmes l'importance de la sécrétion, sans "erreur » possible, opérer euxmêmes les traites ou mulsions et les prélèvements, selon les règles sûres que nous exposons plus loin.

Ceci, dans la pratique, revient à dire que la nourrice doit être sous leur surveillance constante et absolue.

Il suffit de réfléchir un peu, pour se rendre compte combien on rencontre pratiquement de difficultés pour réaliser de telles conditions d'expérimentation.

De fait, il est fort difficile - pour ainsi dire impossible - de trouver un sujet se pliant à une discipline aussi rigoureuse.

20 Difficultés d'ordre social. - Ce sont les premières auxquelles on se heurte dans la recherche des sujets.

Disons tout de suite qu'il ne faut pas compter sur les femmes placées dans une situation aisée, car elles n'accepteront jamais de se plier à une contrainte pénible.

Parmi les autres, presque toutes refusent de se prêter à ces sortes d'expériences, pour de multiples raisons. Chez un très grand nombre, c'est une question de discrétion. Elles répugneront à mettre qui que ce soit, fût-ce un médecin, au courant de leur sécrétion lactée, qu'elles considèrent comme un acte de leur vie intime.

Beaucoup, par pudeur ou par pusillanimité, refusent de laisser pratiquer sur elles les manœuvres de traite, bien que ces dernières soient toujours pratiquées par une femme, docteur en médecine, et dans un local interdit à toute autre personne.

D'autres appréhendent, soit une douleur, - qu'elles sont d'ailleurs fort étonnées de ne pas ressentir, - soit un danger quelconque. Cette crainte est encore plus accentuée quand il s'agit de les faire venir dans les laboratoires de la Faculté de Médecine.

Souvent elles redoutent de devenir un "sujet d'expériences ", soupçonnant de dangereuses arrière-pensées de la part de l'expérimentateur. 
Peut-être, par ailleurs, attribuent-elles un caractère humiliant au fait de se prêter à des expériences qui, à leurs yeux, les assimilent à des femelles animales.

Le qu'en dira-t-on les préoccupe extrêmement. Elles demanderont toujours à être assurées de la plus absolue discrétion, bien que, par ailleurs, elles exigent une large rémunération, peu en harmonie avec de tels scrupules.

A toutes ces sources de difficultés morales relevant de la personnalité même de la femme, s'ajoutent encore, pour celles d'entre elles qui seraient consentantes, les obstacles soulevés par leur entourage.

D'une façon pour ainsi dire générale, toute femme placée sous une influence masculine directe (mari ou amant) ne peut se prêter à ces expériences, bien qu'elles aient lieu dans les conditions de moralité exprimées plus haut. La jalousie de l'homme s'y oppose absolument, même si le ménage souffre de la misère. L'influence des mères n'est pas plus favorable, car elles sont souvent inspiratrices des plus stupides objections et des craintes les moins fondées.

Enfin, ces obstacles moraux surmontés par quelques femmes, celles-ci refusent encore de donner leur lait, dans la crainte très louable que l'alimentation de leur nourrisson n'en pâtisse.

Nous verrons tout à l'heure quel soin nous avons pris pour qu'il n'en soit pas ainsi.

A tous ces empêchements, viennent encore s'ajouter les difficultés créées par la condition sociale du sujet. Des expériences complètes nécessitent chaque jour pour la nourrice une perte de temps considérable, peu compatible avec la vie familiale et les soins du ménage, et à plus forte raison, avec l'exercice d'une profession. Il est évident qu'une femme qui travaille ou qui a plusieurs enfants, ne peut guère s'astreindre huit fois par jour à des mesures de quantité et à des prélèvements délicats.

Sans doute, pour remédier au manque de sujets dans la vie sociale courante, peut-on songer aux milieux hospitaliers, qui semblent plus favorables à ces recherches.

Les nourrices y sont nombreuses et disciplinées ; il n'en est pas moins vrai, pourtant, que les conditions d'expérimentation n'y sont cependant pas parfaites.

Dans les maternités, le séjour des accouchées est généralement très court et n'excède guère 15 jours, délai pendant lequel le régime régulier de la lactation n'est pas encore établi.

Dans les infirmeries des maternités (phlébites, suites de couches difficiles, etc.) on peut rarement étudier des cas normaux; et l'état des malades s'oppose à de telles recherches.

Dans les erèches et les nourriceries où, - ce qui est capital, - 
les nourrices sont toujours avec leurs enfants, un contrôle rigoureux reste souvent illusoire. Quel que soit le dévouement du personnel, il est très difficile d'en obtenir, d'une façon constante, des pesées et prélèvements exacts. En effet, leur mentalité professionnelle et le surmenage qui est de règle dans la plupart de ces services sont peu favorables au développement de l'esprit de minutie nécessaire aux recherches.

Comment empêcher, par exemple, dans une salle obseure, où dorment vingt mères et leurs nourrissons, que quelques-unes donnent le sein en cachette à leurs enfants, quand ceux-ci se réveillent la nuit et commencent à crier ? - Peut-on penser franchement qu'il soit l'heure de faire des mensurations longues et précises, qui soulèveraient vite des protestations générales ? - Le personnel le plus dévoué avoue que "la nuit échappe à tout contrôle ".

30 Difficultés d'ordre personnel. - La nourrice, - que n'ont pas arrêtée les considérations ci-dessus, - ne sera pourtant que rarement utilisable pour nos recherches.

En effet :

Elle doit d'abord être docile, et suivre à la lettre les instructions qu'on lui donne.

Elle devra surtout être sincère et véridique ; ne pas affirmer, par exemple, qu'elle n'a pas donné le sein depuis trois heures, alors que 20 minutes avant elle a fait téter son enfant pour l'empêcher de crier.

Il lui faut, en outre, de la patience et de l'exactitude, de l'attention et de la minutie. Il est vite fastidieux, et bientôt très désagréable, d'être obligé toutes les 2 ou 3 heures, souvent la nuit, de faire, et avec précision, des pesées, des mesures, des échantillonnages. Disons, en outre, qu'il est bien rare que la femme soit capable de le faire elle-même correctement.

En somme, il faut qu'elle participe à la conscience du chercheur, comprenne l'intérêt du travail et désire sincèrement y apporter une collaboration.

$4^{\circ}$ Difficultés d'ordre anatomique et d'ordre physiologique. - On devra encore éliminer les quelques-unes des rares nourrices qu'on aura pu trouver, pour les raisons suivantes :

Chez certaines, le prélèvement est rendu très malaisé, ou même impossible, par la forme défectueuse du mamelon, impropre à la traite manuelle (que nous considérons comme bien préférable aux meilleurs tire-lait).

Chez d'autres, la timidité, ou la sensibilité aux moindres contrariétés, est telle qu'il en résulte une inhibition presque absolue de la sécrétion.

Ainsi, nous n'avons pu obtenir qu'une quantité infime de lait 
chez une femme dont l'enfant criait sans cesse pendant la durée du prélèvement.

\section{LES SUJETS D'EXPÉRIENCE.}

Après l'énumération de ces difficultés de tout ordre, il semblerait impossible de trouver un sujet convenable.

a) Par chance, cependant, il s'est trouvé que l'un de nous, Mme C. V., était précisément au début de cette recherche, dans les conditions les meilleures pour l'exécuter avec toute la rigueur désirable en devenant elle-même le sujet d'expériences souhaité.

Accouchée en mai 1931, d'un troisième enfant, qui fut nourri au sein complètement depuis sa naissance, elle était à son septième mois de lactation quand ont commencé ces recherches, qui furent poursuivies chaque jour pendant 9 mois.

Tiré à chaque mulsion en totalité, le lait était recueilli dans des flacons aseptiques gradués, puis mesuré et mélangé. Un échantillon était prélevé pour l'analyse et le reste donné immédiatement à l'enfant.

Cette manœuvre était répétée à l'heure de tous les repas du nourrisson. On pouvait done contrôler ainsi et analyser la totalité de la sécrétion lactée.

Dans ce cas idéal, toutes les objections et difficultés exprimées ci-dessus tombaient d'elles-mêmes, et la discipline nécessaire, si pénible fût-elle, - était d'autant mieux acceptée que la mère, à la fois expérimentateur et sujet, l'avait fixée elle-même.

b) Ce cas, quoique parfait, était évidemment unique. Nous avons voulu, bien entendu, élargir les résultats obtenus, par des recherches sur d'autres nourrices. Ce fut très difficile. Nous devons à l'obligeance du Professeur agrégé RENTHER, d'avoir trouvé à la Nourricerie départementale du Rhône une femme qui nourrissait son enfant dans des conditions tout à fait exceptionnelles. En effet, contrairement au règlement habituel, le nourrisson ne passait pas la nuit auprès de sa mère. Nous avons done pu obtenir une mesure exacte de la quantité de lait par jour, mais les nécessités générales du service ne nous permirent de faire qu'un seul dosage quotidien des matières grasses.

c) Nous avons pu trouver aussi, par les soins des Visiteuses de l'Enfance, une jeune femme qui avait sevré son enfant depuis 48 heures. Grâce à une très forte rémunération, elle consentait à venir chaque jour au laboratoire se faire tirer son lait.

Une seule mulsion suffisait à donner la totalité du lait produit en 24 heures. Nous lui avons ainsi maintenu sa sécrétion et nous avons 
pu observer et examiner là aussi, mais dans des circonstances différentes, la totalité du lait sécrété.

d) En outre, plusieurs nourrices chez lesquelles il était impossible, pour diverses raisons, de connaître d'une façon suffisamment rigoureuse la quantité journalière de lait, nous ont cependant permis de faire toute une série d'observations qui ont étayé, éclairé et complété les résultats que nous avions déjà obtenus.

\section{CONDITIONS TECHNIQUES DE LA RECHERCHE.}

Elles résident à la fois dans les conditions générales de vie de la nourrice, dans la prise de l'échantillon et dans les méthodes de dosage.

a) Conditions de vie de la nourrice. - La nourrice jouissant d'une santé normale, sera maintenue à un régime alimentaire bien équilibré, d'abondance moyenne et de composition variée.

Ce serait une très grosse erreur, sous prétexte de constance dans l'alimentation, d'instituer un régime trop uniforme, par là même anormal, dont le premier résultat serait d'entraîner rapidement la satiété et l'anorexie.

On évitera les excès de fatigue et, si possible, toute contrariété, car l'influence des facteurs psychiques sur la sécrétion lactée est certaine.

b) Contrôle de la quantité de lait sécrétée. - Les meilleures conditions sont celles de la traite totale des deux seins, que nous exposerons au paragraphe suivant, le lait étant recueilli et mesuré aseptiquement, pour permettre de l'utiliser ultérieurement pour l'alimentation de l'enfant.

Dans l'immense majorité des cas, cette manière d'opérèr se révèle impossible, pour les raisons que nous avons données précédemment. Dans ce cas, l'enfant prenant directement le sein sera pesé avec soin avant et après chaque tétée, sans en excepter aucune. Bien qu'on ne puisse contrôler ainsi exactement la quantité émise à chaque mulsion, - car le sein dans cette manière d'opérer ne se vide pas complètement, - on obtiendra ainsi, et par addition du poids de chacune des tétées, la quantité journalière véritablement sécrétée.

Enfin, nous avons dû parfois, pour ménager à l'enfant une nourriture suffisante, employer les deux méthodes à la fois. Le lait d'un des seins était tiré manuellement, mesuré et dosé. Par ailleurs, I'enfant, préalablement pesé, prenait le lait de l'autre sein ; on le repesait ensuite et on s'assurait de l'épuisement du sein, après la tétée, en recueillant au besoin le reliquat du lait, par une traite manuelle de contrôle.

c) Echantillonnage. - C'est là le point capital, au sujet duquel on ne saurait s'entourer de trop de précautions et de minutie. 
1. On opérera toujours à des heures identiques, déterminées selon les cas, soit par une seule prise quotidienne à heure fixe, soit par des prises multiples aux heures "réglées » de l'alimentation habituelle du nourrisson.

2. Pratique de la mulsion. - Nous avons, après de nombreux essais, préféré aux divers modèles de tire-lait utilisés couramment (par succion, pompe à main ou même aspiration électrique rythmée), l'évacuation de la glande mammaire par le procédé manuel, lequel présente de nombreux avantages : sa simplicité, son débit abondant, le fait qu'aucun appareil inconnu n'inquiète la nourrice, et, aussi, parce qu'elle seule permet à la personne qui pratique ces manceuvres un ensemble de notions tactiles fort utiles à la conduite de la traite.

Nous opérons habituellement par légère pression du mamelon et surtout de la région aréolaire entre le pouce et l'index, mancuvre aussi régulièrement rythmée que possible, par pressions ni trop lentes ni trop rapides, au nombre moyen de 60 à 80 par minute. Quand l'épuisement de la glande est assez avancé, ou s'il y a arrêt temporaire de l'écoulement du lait, on obtient souvent de bons résultats en malaxant délicatement la masse même de la glande.

En aucun cas on ne doit agir avec force et brusquerie.

Ce procédé est d'ailleurs analogue à celui qu'on emploie pour la traite des animaux domestiques.

3. La mulsion doit surtout être complète, c'est-à-dire que le sein doit être vidé aussi complètement que possible.

Pour cela, il faut pratiquer la traite à fond, jusqu'au moment où l'on ne peut plus obtenir de lait et où la glande change de consistance, perdant sa ferme tension initiale, pour devenir molle et dépressible. A signaler, ici, que cet épuisement complet du lait sécrété n'est pas toujours aisé à reconnaître. Les sensations pénibles et douloureuses, les influences psychiques, les moindres distractions de la nourrice peuvent amener des inhibitions momentanées qui font croire que la glande est épuisée, alors que cela n'est pas, et que la sécrétion donnera encore des quantités importantes.

Il importe donc que ces manœuvres soient pratiquées, non seulement avec une extrême douceur et beaucoup d'habileté, mais encore avec une grande patience et une longue habitude. Seule, peut-être, une femme qui a tiré elle-même son lait d'une façon coutumière, - comme ce fut le cas pour ces recherches, - aura le doigté voulu pour amener, dans tous les cas, la mulsion complète.

4. Il faut vider de cette façon chacun des deux seins et recueillir le lait séparément dans deux flacons gradués stérilisés. Chacun de ces flacons sera agité pour rendre le lait homogène, et une partie aliquote en sera prélevée pour les dosages comparatifs du lait de 
chaque sein. Si au contraire on a besoin d'un seul dosage du lait total des deux seins, les deux flacons seront mélangés en un seul, que l'on agitera avec soin de nouveau avant prélèvement.

Si enfin, on veut faire des dosages fractionnels dans chacun des deux seins, le lait sera recueilli $10 \mathrm{~cm}^{3}$ par $10 \mathrm{~cm}^{3}$ dans une série d'éprouvettes graduées. La sécrétion totale sera donnée par l'addition des différents volumes partiels obtenus. Avec un peu d'habitude, on peut même pratiquer la traite simultanée des deux seins, laquelle est nécessaire dans certains cas, comme nous le verrons plus loin.

5. Les débits seront notés aussitôt et les prélèvements effectués analysés aussitôt que possible.

Malgré l'emploi des installations frigorifiques, il y a le plus grand intérêt à agir vite, en l'espèce avant que l'émulsion naturelle du lait ne soit troublée.

Dans les cas exceptionnels où l'analyse doit être retardée, il faut éviter de trop agiter le lait, lequel est à ce moment séparé en plusieurs couches.

Dans le cas du lait de femme, une agitation légère suffit pour l'homogénéiser à nouveau, alors qu'un brassage énergique risque de barrater le beurre le long des parois du vase.

d) Méthode de dosage. - Nous avons utilisé le procédé courant d'ADAM : traitement du lait par le mélange éthéroalcoolique-ammoniacal et précipitation de la caséine par l'acide acétique dilué.

Le dosage portait sur $10 \mathrm{~cm}^{3}$. La solution terminale éthérée de beurre était recueillie dans une petite capsule tarée ; l'appareil était rincé par agitation avec de l'éther, et cet éther de lavage joint à la solution de beurre dans la capsule tarée. Celle-ei, après évaporation de l'éther à l'air libre, était mise à l'étuve à $100^{\circ}$ pendant 6 heures, avant d'être pesée à nouveau.

IV. RÉsUltats.

\section{A. RECHERCHES SUR LES VARIATIONS QUANTITATIVES DU LAIT SECRÉTÉ.}

\section{A. RECHERCHES SUR LES VARIATIONS QUANTITATIVES DU} LAIT SÉCRÉTÉ.

L'abondance du lait de la femme est évidemment infiniment variable selon les sujets. Alors que certaines nourrices ne donnent que quelques grammes par jour, on a pu observer des quantités quotidiennes très élevées.

Dans cet ordre d'idées, Kollmann cite le cas d'une domestique qui à partir du $62^{\mathrm{e}}$ jour de lactation a fourni - et pendant un an une moyenne journalière de 5.900 gr. de lait. Elle nourrissait jusqu'à einq enfants en même temps. 
a) modus operandi. - Nous avons dit qu'on obtenait la mesure exacte de la quantité de lait sécrétée, soit par traite manuelle, soit par pesée du nourrisson avant et après chaque tétée dans des conditions précisées plus haut

Nous avons pu vérifier la variabilité considérable des quantités de lait sécrétées, déjà signalée par d'autres auteurs. Nous avons suivi la sécrétion à chacune de ses manifestations pendant des mois (Cf. courbes A, B, C, E, ligne supérieure) et pu constater un certain nombre de faits assez constants.

b) DÉbits journaliers (périodes de 24 heures). - Si l'on a sous les yeux des courbes de quantité journalière de lait, pour une nourrice donnée, ce qui frappe immédiatement, c'est la fréquence et l'importance des oscillations. Celles-ci, au premier abord, sont complètement irrégulières. Cependant, si on examine avec beaucoup de soin les courbes, on peut apercevoir certaines phases de moindres variations. Elles sont suivies d'ailleurs de périodes pendant lesquelles les différences quantitatives sont plus accentuées et présentent, d'un jour à l'autre, des points extrêmes très éloignés.

Même chez certaines nourrices en pleine lactation, on constate que les périodes de fixité relative de la quantité de lait sécrétée peuvent atteindre une durée de 5 ou 6 jours (courbe $\mathrm{C}$ ). Cette fixité est plus apparente encore lorsqu'on a affaire à des nourrices dont la sécrétion est peu abondante (lactation de sevrage ou lactation maintenue artificiellement basse) ( $C f$. courbes D, E et F). Par ailleurs, dans une lactation normale (par "lactation normale" nous entendons qu'il ne s'agit ni de diminution ni d'intensification du nourrissage, et de conditions d'existence régulière), dans une lactation normale, disons-nous, les périodes de variabilité, quoique montrant à la courbe des points extrêmes, bas ou élevés, offriraient cependant une constance relative si on établissait des moyennes pour des périodes de 4 à 5 jours.

On peut observer aussi, d'une façon presque constante, que lorsque la quantité de lait est très élevée un jour, celle du lendemain est généralement particulièrement minime.

D'autre part, pendant les périodes de diminution ou d'intensification du nourrissage, les variations d'un jour à l'autre, généralement réduites, sont telles que l'allure de la courbe devient le plus souvent, soit descendante, soit ascendante (courbes D et F).

Nous avons pu encore observer des cas de lactation brusquement modifiée.

Une primipare de 18 ans ayant nourri sans peine son enfant pendant 13 jours ( 7 tétées par 24 heures), le sèvre brusquement. Nous avons pu la voir 48 heures après, en pleine période de rétention. 


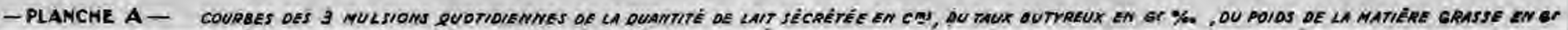

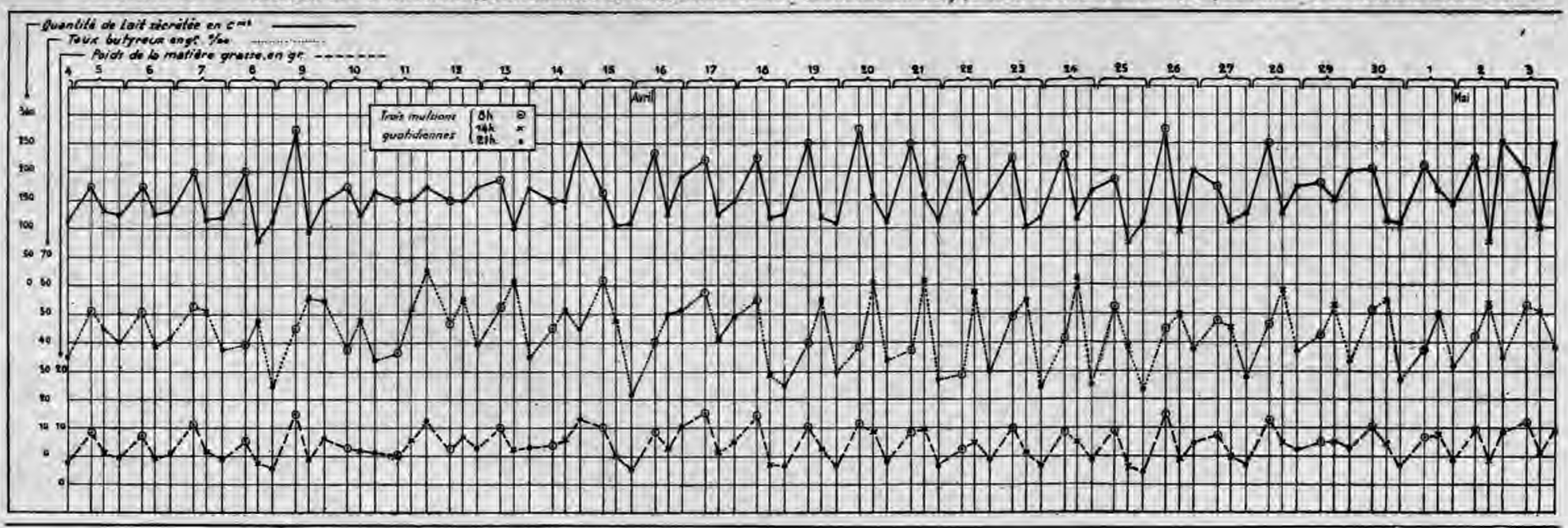

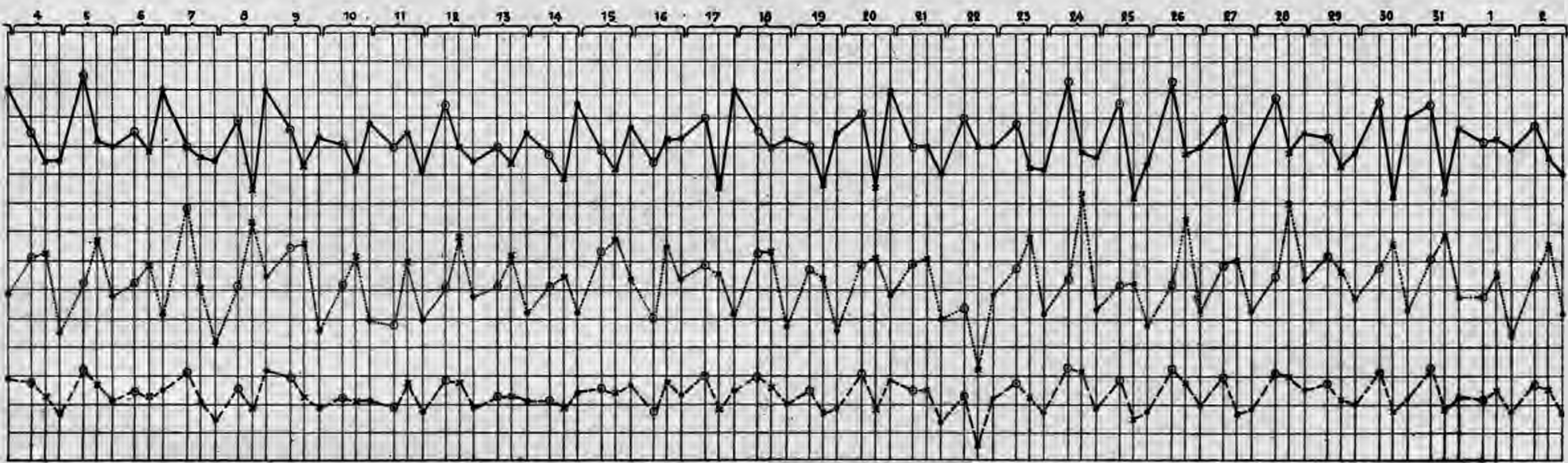

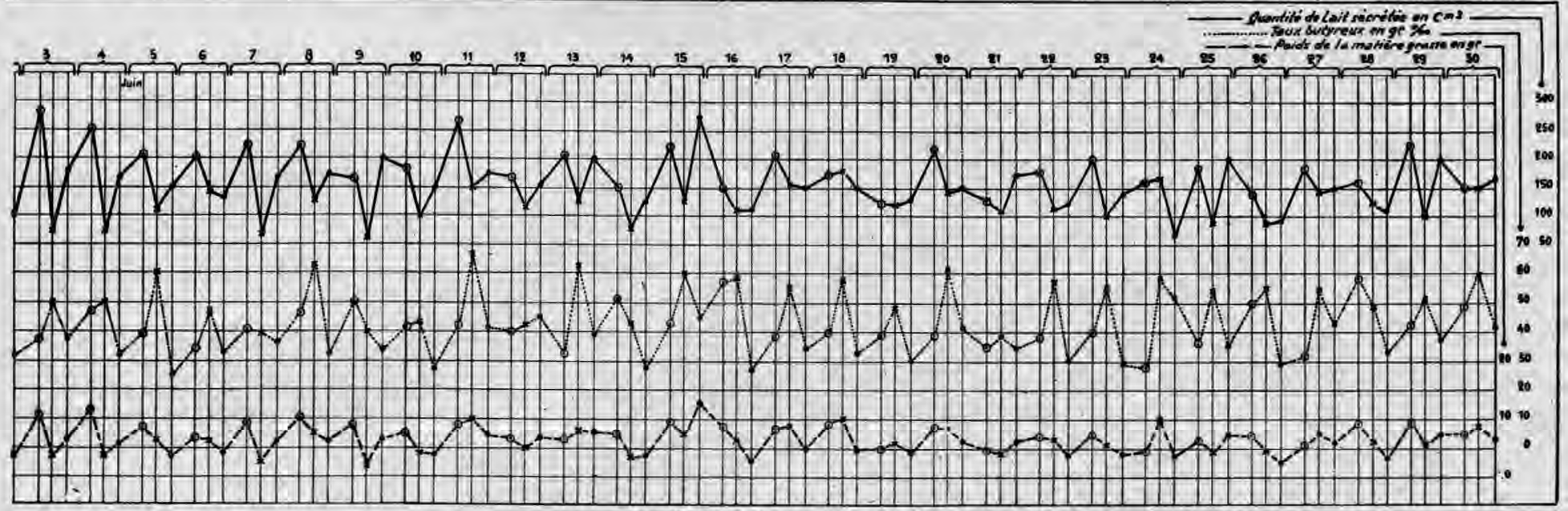

PLANCHE A. - Observations $M^{\text {me }}$ C. $\boldsymbol{V}$.

Courbes des trois mulsions quotidiennes :

I0 de la quantité de lait séerété, en centimétres cubes. -

20 du taux butyreux, en grammes pour 1.000

$3^{\circ}$ du poids de matière grasse, en grammes ....... 
- PLANCHE B - COURBE JOURNALIERE DU TOTAL DES 3 MULSIONS QUOTIDIENNES POUR.

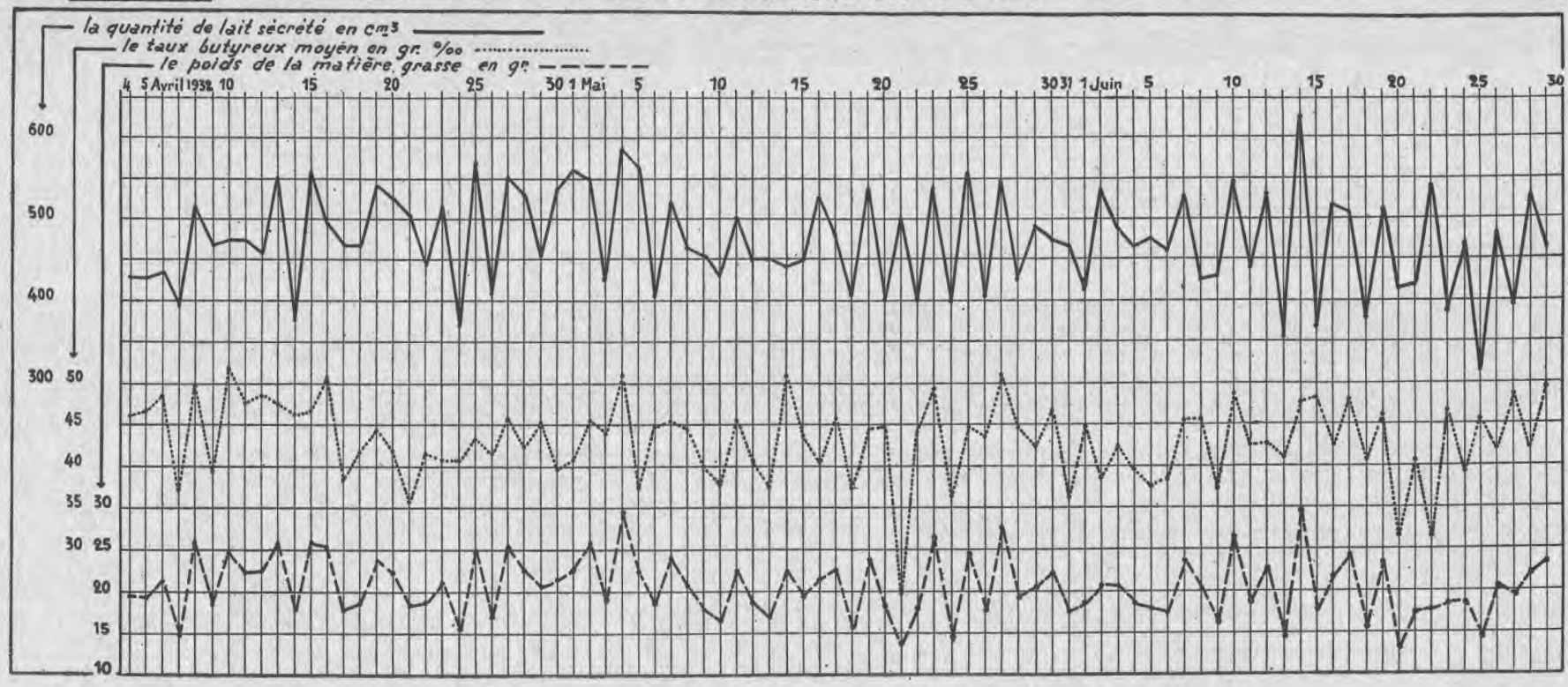

PLANCHE B. - Observation $C . V$.

Courbes du total des trois mulsions quotidiennes pour :

$1^{\circ}$ la quantité de lait sécrété, en centimètres cubes

$2^{\circ}$ le taux butyreux moyen, en grammes pour 1.000

$3^{\circ}$ le poids de la matière grasse, en grammes ...... 


\section{-PLANCHE E-REPRISE DE LACTATION PAR UME SEULE MULSION QUOTIDIENME APRES UNE RÉTENTION}

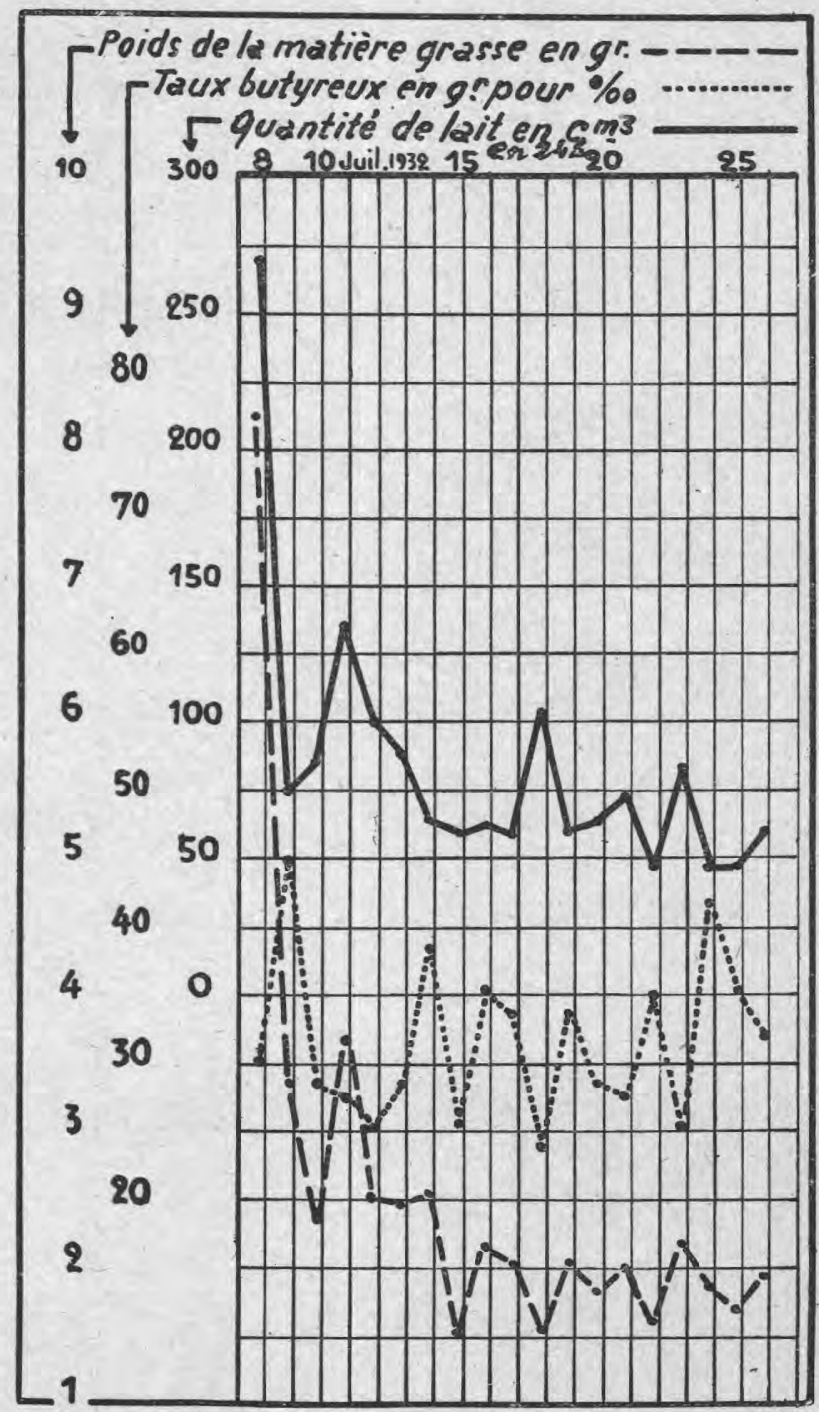

PLANCHE E. - Observation Mile $R$.

Les seins, très distendus, durs et douloureux, laissaient de temps à autre s'écouler spontanément du lait en quantité notable, mais difficile à préciser. La mulsion, qui fut fort délicate à pratiquer, donna 270 gr., soulageant immédiatement le sujet. Le lendemain, à 
la même heure, on obtenait seulement $75 \mathrm{gr}$,, et au bout de 7 jours, après quelques variations, la quantité journalière obtenue par une seule traite restait au voisinage de $60 \mathrm{gr}$., sans aucun phénomène de rétention, et se poursuivait ainsi pendant une vingtaine de jours, jusqu'à la cessation volontaire de l'observation (courbe E).

(A suivre.)

\title{
SUR L'INFLUENCE DE DIFFÉRENTS FACTEURS SUR LA TENDANCE A LA FERIMENTATION GAZEUSE PRÉCOCE DU FROMAGE
}

\author{
par \\ E. HAGLUND, E. SANDBERG et Chr. BARTHEL. \\ (Travail des Sections de laiterie et de bactériologie de l'Institut central \\ de Recherches agronomiques à Stockholm.)
}

Dans quelques travaux des années 1928-1930 de la Section de laiterie de l'Institut central de Recherehes agronomiques, nous avons traité la question de l'influence de la méthode de fabrication sur les transformations biochimiques et sur la consistance du fromage. De ces travaux, il ressort que la manière de saler et la quantité de sel ajoutée [1] ont une influence marquée sur le nombre bactérien et sur la rapidité de maturation du fromage, et, ensuite, que la consistance de celui-ci dépend en premier lieu du $p$ H à la fin de la fermentation lactique [2]. Un $p H$ élevé dans le fromage frais détermine une consistance souple et une pâte facile à couper, tandis que le fromage devient friable et de pâte courte à un $p$ H trop bas. Un $p H$ élevé est obtenu en premier lieu en employant un lait relativement pauvre en-bactéries lactiques. La fermentation lactique devient alors lente, la perte de chaux est peu considérable [3] et le fromage prend un $p H$ relativement élevé et une consistance souple.

La consistance des fromages à pâte dure a fortement attiré l'attention au cours de ces dernières années. Les efforts pour augmenter la consommation du fromage dans le pays a eu comme résultat que les exigences sont devenues beaucoup plus grandes en ce qui eoncerne sa consistance. Les laiteries cherchent donc, par des moyens techniques, à donner au fromage la consistance désirée. Cela amène une élévation du $p H$, et la question se pose alors de savoir si cette eirconstance augmente considérablement la tendance à la fermentation gazeuse précoce du fromage. Cette question fut rendue actuelle du fait qu'une laiterie demanda à la Section laitière de l'Institut de Recherches agronomiques d'essayer de trouver les causes de la fermentation gazeuse précoce que subit constamment le fromage de cette laiterie pendant et immédiatement après la mise sous presse. 